\title{
Evidence of a Spatial Encoding Deficit in Rats with Lesions of the Mammillary Bodies or Mammillothalamic Tract
}

\author{
Seralynne D. Vann and John P. Aggleton \\ School of Psychology, Cardiff University, Cardiff, CF10 3YG, United Kingdom
}

The present study sought to identify the role of the mammillary bodies and their projections to the anterior thalamic nuclei for spatial memory. Rats with either selective, neurotoxic mammillary body lesions or discrete mammillothalamic tract lesions were tested on various spatial working memory tasks. Tests using the T-maze, radial-arm maze, and water maze were manipulated to compare three possible theories of mammillary body function by increasing proactive interference, increasing retention interval, and taxing the rapid processing of novel spatial stimuli. On T-maze alternation and radial-arm maze tasks, both lesion groups were initially impaired but seemed to recover. Transfer tests revealed, however, a more permanent change in performance, suggesting a failure to use distal (allocentric) cues. Consistent with this, both groups were also impaired at matching-to-place in the water maze and showed little improvement with practice. Nevertheless, once the lesion groups had been trained on a task, they were not affected differentially either by an increase of proactive interference or by retention intervals of up to $30 \mathrm{~min}$. Although both mammillary body and mammillothalamic tract lesions resulted in similar impairments, the mammillothalamic tract group was the more affected when acquiring new spatial information. Together, these results suggest that mammillary body damage causes an encoding deficit when learning new spatial tasks, resulting in a suboptimal mode of performance, which may reflect a loss of directional heading information.

Key words: amnesia; anterior thalamic nuclei; Delay and Brion circuit; mammillary bodies; mammillothalamic tract; rat; spatial memory

\section{Introduction}

The mammillary bodies (MBs) have long been considered to play an important role in memory (Delay and Brion, 1969; Sziklas and Petrides, 1998). Clinical evidence, ranging from the diffuse pathology of the Wernicke-Korsakoff syndrome (Gamper, 1928; Victor et al., 1989) to the more discrete pathology associated with tumors and traumatic injury (Dusoir et al., 1990; Tanaka et al., 1997; Hildebrandt et al., 2001), has repeatedly implicated this region. The anatomical connections of this structure are consistent with such a role because MBs receive a major input from the hippocampus, via the fornix, whereas their main output is to the anterior thalamic nuclei, via the mammillothalamic tract (MTT) (Cruce, 1975; Watanabe and Kawana, 1980; Seki and Zyo, 1984; Allen and Hopkins, 1990). It has been proposed that these connections form a circuit (the "Delay and Brion" circuit) that links key temporal lobe and diencephalic regions involved in memory (Delay and Brion, 1969; Gaffan, 1992; Aggleton and Brown, 1999).

Animal research testing this proposal has focused primarily on spatial memory. This reflects the importance of the hippocampus for this form of memory (O'Keefe and Nadel, 1978; Morris et al., 1982, 1990). The effects of MB lesions in rats have been varied, however, and this may reflect different surgical techniques. When electrolytic lesions are produced there is the likelihood of damage to adjacent fiber tracts (e.g., the medial forebrain bundle, mammillary peduncle, supramammillary decussation), whereas all lesion techniques risk invading the adjacent supramammillary nuclei (Sutherland and Rodriguez, 1989; Saravis et

Received 0ct. 30, 2002; revised Jan. 30, 2003; accepted Jan. 31, 2003.

This research was partly supported by a Wellcome Trust grant. We thank Heather Phillips for her assistance.

Correspondence should be addressed to Dr. Seralynne Vann, School of Psychology, Cardiff University, Cardiff,

CF103YG, United Kingdom. E-mail: vannsd@cardiff.ac.uk.

Copyright $\odot 2003$ Society for Neuroscience $\quad 0270-6474 / 03 / 233506-09 \$ 15.00 / 0$ al., 1990; Aggleton et al., 1991; Sziklas and Petrides, 1993; Neave et al., 1997). Because these nuclei set hippocampal theta rhythm (Kirk, 1998), their involvement may be an important variable. As a consequence, there remains uncertainty over the selective contribution of MBs.

The MTT is of particular relevance in this regard because it is the only structure with connections confined within the Delay and Brion circuit; therefore, transection of the MTT may be especially informative in understanding the role of this circuit. Surprisingly, very few previous studies have examined MTT lesions in rodents. Furthermore, previous MTT lesions have tended to be extensive, and behavioral studies have essentially been confined to T-maze alternation (Krieckhaus and Randall, 1968; Field et al., 1978; Thomas and Gash, 1985). Given the limited data currently available, the present study sought to compare selective MB lesions with discrete MTT lesions.

Three hypotheses concerning the functions of MB were examined. First, the MBs help to distinguish separate events by enhancing contextual differences, thereby reducing proactive interference (Jaffard et al., 1991; Aggleton et al., 1995). Second, the MBs are important when holding information over extended retention intervals (Saravis et al., 1990; Beracochea and Jaffard, 1995; Sziklas and Petrides, 1998). Third, the Delay and Brion circuit, including MBs, is recruited when there is rapid learning of new spatial landmarks (Vann et al., 2000a,b). Consistent with this, the effects of MB lesions ameliorate with training (Sutherland and Rodriguez, 1989; Aggleton et al., 1995; Neave et al., 1997).

\section{Materials and Methods}

Subjects and surgery

Subjects were 32 male pigmented rats (Dark Agouti strain; Harlan, Bicester, UK) weighing between 217 and $301 \mathrm{gm}$ at the time of surgery. Animals were housed in pairs under diurnal light conditions (14 hr 
light/10 hr dark), and testing was performed during the light phase. Animals were given ad libitum access to water throughout. All experiments were performed in accordance with the UK Animals (Scientific Procedures) Act 1986 and associated guidelines.

Animals were deeply anesthetized by intraperitoneal injection of sodium pentobarbital $(60 \mathrm{mg} / \mathrm{kg}$ ). The 12 rats receiving mammillary body lesions $(\mathrm{MBx})$ were then placed in a stereotaxic headholder (David Kopf Instruments, Tujunga, CA) with the nose bar at +5.0 , and the scalp was cut and retracted to expose the skull. The lesions were made by injecting 0.09 M NMDA (Sigma, Poole, UK) dissolved in phosphate buffer, pH 7.2. Injections were made in one site per hemisphere using a $1 \mu$ l Hamilton syringe. The stereotaxic coordinates of the lesion placements relative to ear-bar zero were anteroposterior (AP) +3.2 and lateral (L) \pm 0.7 , and the depth, from top of cortex, was $-9.3 \mathrm{~mm}$. Bilateral injections of $0.6 \mu \mathrm{l}$ were injected over $8 \mathrm{~min}$, and the needle was then left in situ for $10 \mathrm{~min}$. After the injections of NMDA, animals were injected with $0.05 \mathrm{ml}$ of a respiratory stimulant (Millophyline, Arnolds Veterinary Products, Shrewsbury, UK). At the completion of all surgeries, the skin was sutured, and an antibiotic powder (Acramide; Dales Pharmaceuticals, Skipton, UK) was applied topically. Animals also received subcutaneous injections of $5 \mathrm{ml}$ of glucose and were given paracetamol in their drinking water for $3 \mathrm{~d}$ after surgery. The six animals acting as MBx controls received the same procedure and drugs as the animals receiving lesions, which involved the needle being lowered into the same coordinates and the injection of the same volume of saline.

For the eight animals receiving mammillothalamic tract lesions (MTTx), the animals received a procedure similar to the MB animals but the lesions were made by radio frequency using a Radionics TCZ (Radionics, Burlington, VT) electrode ( $0.3 \mathrm{~mm}$ tip length, $0.25 \mathrm{~mm}$ diameter). This was lowered vertically into the mammillothalamic tract, and the tip temperature was raised to $70^{\circ} \mathrm{C}$ for 50 sec using an RFG4-A Lesion Maker (Radionics). One lesion was made in each hemisphere. The stereotaxic coordinates of the lesion relative to ear-bar zero were AP +4.2 and $\mathrm{L}$ \pm 0.9 , and the depth from top of cortex was $-6.9 \mathrm{~mm}$. At the completion of all surgeries, the skin was sutured and an antibiotic powder (Acramide; Dales Pharmaceuticals) was applied, and animals received a $5 \mathrm{ml}$ injection of glucose saline and analgesia (Temgesic, Reckett and Colman). The four animals acting as MTT surgical controls received the same procedure and drugs as the animals receiving lesions, which involved the probe being lowered to a slightly higher depth than that used for the lesions.

\section{Apparatus and behavioral training}

Experiment 1: reinforced spatial alternation in the T-maze. Testing was performed in a modifiable four-arm (cross-shaped) maze. The four arms (70 cm long, $10 \mathrm{~cm}$ wide) were made of wood, and the walls (17 cm high) were made of clear Perspex. At any time one of the arms could be blocked off to form a T-shaped maze. Aluminum barriers could be positioned $\sim 25 \mathrm{~cm}$ from the end of each arm to create a start area. The maze was supported by two stands $(94 \mathrm{~cm}$ high) and was situated in a rectangular room $(280 \times 300 \times 240 \mathrm{~cm})$ with salient visual cues.

Testing began at least 2 weeks after surgery, by which time the rats had regained their preoperative weight. Animals were subsequently foodrestricted to $85 \%$ of their free-feeding body weight, although water remained available ad libitum. Each animal was given $7 \mathrm{~d}$ of $5 \mathrm{~min}$ pretraining to train them to run reliably down the stem of the maze and find food pellets in the food wells in both arms. After this the acquisition phase began.

At the start of each acquisition trial, which consisted of two stages, two food pellets (45 mg; Noyes Purified Rodent Diet) were placed in each food well, and an aluminum block was placed at the neck of the T-maze, thereby closing off one arm. As a consequence, on each "sample run" the animal was forced to enter the open arm where it was allowed to eat the food at the end of the arm. The animal was then picked up and confined in the start box for a delay of $10 \mathrm{sec}$, during which time the aluminum block was removed. The door to the start arm was then opened, and the animal was allowed a free choice between the two arms of the T-maze. On this "choice run" the animal was considered to have chosen the correct arm if it had alternated, i.e., had entered the arm not previously entered on the sample run, and would then be allowed to eat the food reward before being returned to the holding box. If the animal made an incorrect choice, i.e., returned to the arm visited on the sample run, the rat was confined to that arm for $\sim 5 \mathrm{sec}$ before being returned to the traveling box. The rats were tested in groups of four, with each animal having one trial in turn so that the intertrial interval (ITI) was $\sim 4 \mathrm{~min}$. The animals received six trials per day for a total $12 \mathrm{~d}$.

The acquisition phase was immediately followed by eight sessions on a test of continuous alternation. At the start of each of these sessions the rat was forced to enter either the right or left arm, and this initial sample run was rewarded with two pellets. This sample run was immediately followed by 10 consecutive massed trials in which the correct choice was always the arm opposite to the one chosen on the previous trial. The ITI was $15 \mathrm{sec}$. There were no correction trials.

This was followed by a final four continuous alternation sessions, each of 12 trials. Three different retention intervals (i.e., the time spent in the start box before the test run) of 10,20, or $40 \mathrm{sec}$ were used, and an equal number of each of these delays was used each day. In this way the animals received a total of 16 trials at each delay.

Experiment 2: radial-arm maze. Testing was performed in an eight-arm radial maze. The maze consisted of an octagonal central platform $(34 \mathrm{~cm}$ diameter) with eight equally spaced radial arms $(87 \mathrm{~cm}$ long, $10 \mathrm{~cm}$ wide). The floor of the central platform and the floors of the eight arms were made of wood, and clear Perspex ( $24 \mathrm{~cm}$ high) formed the walls of the arms. Close to the farthest end of each arm was a food well $(2 \mathrm{~cm}$ in diameter and $0.5 \mathrm{~cm}$ deep). At the start of each arm was a clear Perspex guillotine door (12 cm high) that controlled access in and out of the central platform. Each door was attached by a pulley system enabling the experimenter to control access to the arms. The maze could be positioned in either of two rooms $(295 \times 295 \times 260 \mathrm{~cm}$ or $255 \times 330 \times 260 \mathrm{~cm})$, both of which contained salient visual cues such as geometric shapes and high contrast stimuli on the walls. These rooms were different from that used in experiment 1, and the appearance of the two rooms in experiment 2 was markedly different (different size, different shape, and different visual cues on walls).

Animals were maintained on restricted feeding at $85 \%$ of their freefeeding body weight. Pretraining for the radial-arm maze began after the completion of testing in the T-maze and involved one habituation session in which the animals were allowed to explore the maze freely for 5 min with the guillotine doors raised and food pellets ( $45 \mathrm{mg}$; Noyes Purified Rodent Diet) scattered down the arms. The animals were then trained on the standard radial-arm maze task (see below). A time limit of 10 min was placed on each trial (visiting eight different arms), so that trials lasting longer were terminated and not regarded as complete trials. Formal training lasted for 27 sessions and consisted of several stages.

Stage 1 (sessions 1-14) was the standard working memory version of the radial-arm maze task (Olton et al., 1978) in which the animals' optimal strategy was to retrieve the reward pellets from all eight arms without reentering any previously entered arms. At the start of a trial, all eight arms were baited with two food pellets. The animal would make an arm choice and then return to the central platform, and all the doors were closed for $\sim 10 \mathrm{sec}$ before they were opened again, permitting the animal to make another choice. This continued until all eight arms had been visited (i.e., a complete trial within $10 \mathrm{~min}$ ). The number of sequential choice responses was calculated, which was when the animals' successive choices involved immediately adjacent arms in a constant direction. It was measured by giving the animal a score of +1 (clockwise) or -1 (anti-clockwise) if the arm was immediately adjacent to previous choice and 0 for any other arm choice. A higher absolute score would therefore reflect the use of a sequential response strategy (Olton and Samuelson, 1976; Ennaceur and Aggleton, 1997).

Stage 2 (sessions 15-18) was to test for the possible use of intra-maze cues in performing the task. The start of the session was as before, but after the animal had made four different arm choices, it was removed from the maze. The animal was placed in a traveling box, which had an aluminum top, base, and sides $(10 \times 10 \times 26 \mathrm{~cm})$, that was also in the testing room. The maze was then rotated by $45^{\circ}$, and the remaining food pellets were moved so that they were still in the same allocentric locations but the actual arms had changed. The animal was then returned to the 
central platform after the $60 \mathrm{sec}$ that it took to rotate the maze, and the session continued until all reward pellets had been retrieved.

Stage 3 (sessions 19-22) was the same as the above stage: the maze was rotated after the animal had made four arm choices, but it also involved a 20 min delay between the first four choices and the animal being returned to the maze.

Stage 4 (sessions 21-24) was to increase task difficulty by increasing the degree of proactive interference. This was achieved by giving the animals two consecutive trials each day, with a 2 min ITI. In all other respects the training was the same as Stage 1.

Stage 5 (session 25) was to determine the effects of novel environmental cues on the animals' performance. For the final day (session 25), the animals were run in the same maze that had been used throughout, but this time it was placed in a novel room with different spatial landmarks (see Apparatus and learning behavior). Animals were given two trials, again with a 2 min ITI.

Experiment 3: water maze. Two water mazes were used (both $200 \mathrm{~cm}$ diameter, $60 \mathrm{~cm}$ deep, and made of white fiberglass and mounted $58 \mathrm{~cm}$ above the floor). The pools were filled with water $\left(24 \pm 1^{\circ} \mathrm{C}\right)$ made opaque by the addition of nontoxic emulsion (Opacifier, Chesham Chemicals, Harrow, UK). An escape platform $(10 \mathrm{~cm}$ diameter, $2 \mathrm{~cm}$ below water surface) could be placed in the pool. One pool was in room $1(305 \times 396 \mathrm{~cm})$, and the other was in room $2(440 \times 400 \mathrm{~cm})$. In both rooms lighting was provided by four floor-mounted spotlights $(500 \mathrm{~W})$. Both rooms contained salient visual cues such as geometric shapes and high-contrast stimuli on the walls. These cues were different in the two rooms. Both rooms had a curtain hanging from the ceiling around the pool that could be opened or closed. Swim paths were tracked with a video camera suspended directly above each pool. Data were collected and analyzed on-line with an HVS image analyzer connected to a computer that used Watermaze Software (Edinburgh, UK).

The same animals were used as for the previous experiment except that one of the animals from the MB group suffered an acute, abdominal aortic aneurysm, leaving $11 \mathrm{MB}$ animals. For this experiment both food and water were available ad libitum.

The animals were trained on a working memory task ("delayed matching-to-place") in water maze room 1 . For this, 12 platform positions, which varied in their distance from the pool perimeter, were used along with eight possible start positions. Animals received $2 \mathrm{~d}$ of pretraining with four swims per day. For this a curtain was drawn closed around the pool, and both the start position and platform position were changed for every forced swim. Each swim was terminated when the animal either located the submerged platform or after $120 \mathrm{sec}$ had elapsed. If the animal had not located the platform at the end of $120 \mathrm{sec}$, it was guided there by the experimenter and then had to remain on the platform for $30 \mathrm{sec}$. The animals were transported between the holding room and water maze in an opaque, aluminum traveling box. They were also placed in the opaque holding box between each trial.

For the $12 \mathrm{~d}$ of actual training the curtain was removed from around the pool, i.e., room cues were visible. The location of the platform remained constant across the four trials of a given day but varied between days. The same start position was used for the first two trials of each session but was then varied for the remaining two trials. This made it possible to match distances for trials 1 and 2, the key comparison. Each trial terminated when the animal had either located the platform or $120 \mathrm{sec}$ had elapsed. The animals were then left on the platform for $30 \mathrm{sec}$. The next trial began almost immediately afterward, giving an ITI of $\sim 15 \mathrm{sec}$.

On days 13-16 the delay between the first and second trials was increased to $30 \mathrm{~min}$, during which time the animal was returned to the home cage. After the second trial the ITI was $\sim 15 \mathrm{sec}$ as before.

On days 17 and 18 the animals received $2 \mathrm{~d}$ of the standard matchingto-place task with a $15 \mathrm{sec}$ ITI for all trials. On days 19 and 20 the animals received a further $2 \mathrm{~d}$ of this task, but on these $2 \mathrm{~d}$ they were tested in water maze room 2 .

\section{Histological procedures}

On completion of the experiments, the animals were deeply anesthetized with sodium pentobarbital $(60 \mathrm{mg} / \mathrm{kg})$ and perfused transcardially with
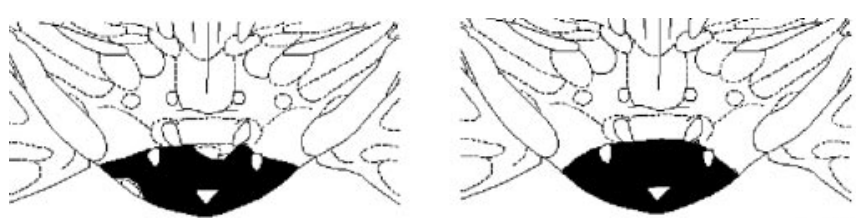

$-4.30$
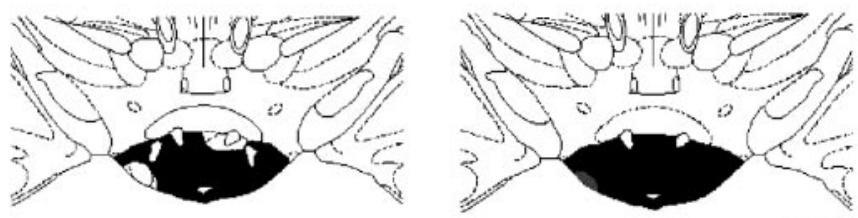

$-4.52$
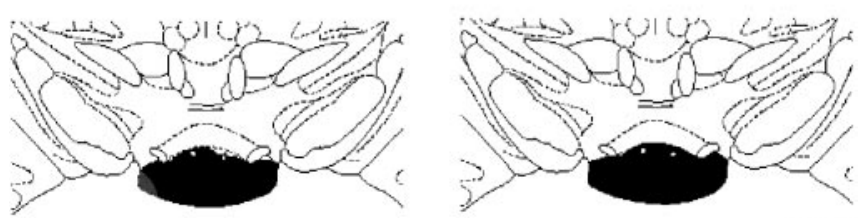

$-4.80$
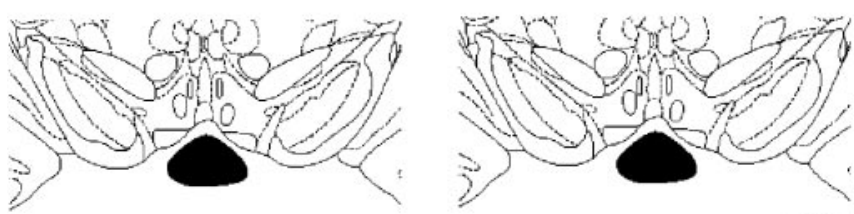

$-5.20$

Figure 1. Diagrammatic reconstructions showing the extent of MB lesions. Areas in black show complete loss of neurons, whereas gray areas show partial cell loss. The left column depicts the smallest of the MB lesions, and the right column depicts the largest. The sections are modified from Paxinos and Watson (1997), and the numbers indicate the distance from bregma.

0.1 M PBS followed by $10 \%$ formol-saline. The brains were removed and postfixed in $10 \%$ formol-saline and then transferred to $25 \%$ sucrose overnight. Coronal sections were cut at $40 \mu \mathrm{m}$ on a freezing microtome, and a one-in-three series of sections was mounted onto gelatin-coated slides and stained with cresyl violet, a Nissl stain.

\section{Results}

\section{Histological analysis}

Mammillary bodies

In all $12 \mathrm{MBx}$ cases the surgery resulted in a circumscribed lesion of the mammillary bodies. In nearly all cases the NMDA injection resulted in almost complete loss of neurons in all of the various mammillary body nuclei. Only one case showed any appreciable cellular sparing, and this was subsequently removed from analyses. The supramammillary nuclei were spared in all cases. The lesions tended to show a pattern of very slight lateral mammillary body sparing on the left hemisphere and slight sparing of the dorsal medial margin of the medial mammillary body in the right hemisphere (Figs. 1, 2). This sparing resulted in only a very few cells looking normal, and the remainder were shrunken and clearly distorted. Cell loss in the posterior nucleus was complete in all cases. The third ventricle was enlarged in all cases. The principal mammillary tract was reduced in size, and the MTT was present more rostrally, although it showed signs of atrophy.

\section{Mammillothalamic tract}

In all but two cases the lesion resulted in a complete bilateral mammillothalamic tract lesion. In these two cases, the lesion was 

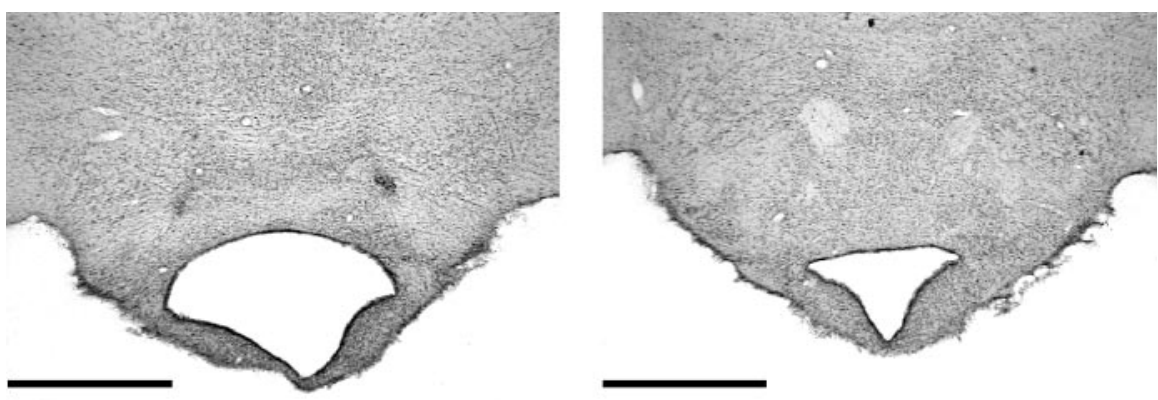

Figure 2. Photomicrograph of coronal sections showing a typical MB lesion on the left and a sham brain on the right. Scale bar, $1 \mathrm{~mm}$.
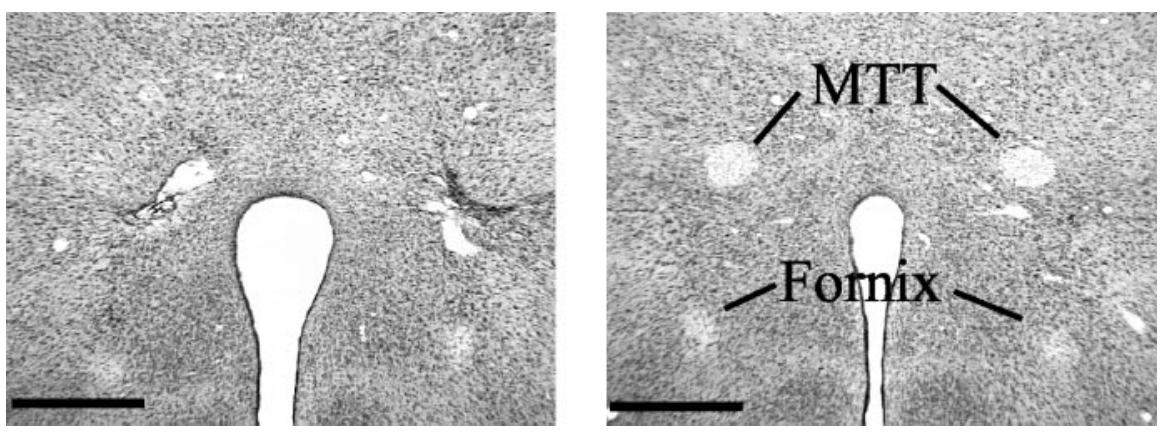

Figure 3. Photomicrograph of coronal sections showing a typical MTT lesion on the left and a sham brain on the right. Scale bar, $0.5 \mathrm{~mm}$.

complete in one hemisphere, but there was partial sparing in the other. These cases were subsequently removed from further analyses. All remaining lesions were highly discrete (Fig. 3), but because of the location of the tract, there was damage immediately adjacent to part of the zona incerta. There was both lateral and medial mammillary body degeneration, although there was a tendency for the medial degeneration to be greater. The placement of the lesions was sufficiently rostral so that there was no direct damage to the mammillary bodies, supramammillary nuclei, or mammillotegmental tract (Fig. 3). In no case was there damage to the postcommissural portion of the fornix (Fig. 3).

The final groups comprised mammillary body lesions (MBx; $n=11$ ), mammillothalamic tract lesions (MTTx; $n=8$ ), and controls made up of four MTTx surgical controls and six MBx surgical controls. The two control subgroups did not differ on any of the tasks (all $p>0.2$ ) and were therefore combined throughout ( $\operatorname{sham;} n=10$ ).

\section{Experiment 1: reinforced spatial alternation in the T-maze}

The initial acquisition stage was divided into six blocks of $2 \mathrm{~d}$. A comparison showed a highly significant group effect $\left(F_{(2,26)}=\right.$ $13.0 ; p<0.001)$, as well as a significant effect of block $\left(F_{(5,130)}=\right.$ $5.4 ; p<0.001)$, reflecting the improvement in performance during task acquisition (Fig. 4). Subsequent comparisons between the scores of the three groups confirmed that both the MBx and MTTx groups were significantly impaired compared with the shams (Newman-Keuls; both $p<0.01$ ) but with not each other. The simple effects showed that the MBx and MTTx groups were impaired for the first four blocks of sessions, but there was no group difference for sessions 9-12.

The animals were then tested for $8 \mathrm{~d}$ (10 trials per day) on the continuous alternation procedure (Fig. 4), which should increase levels of proactive interference. Scores were blocked over $2 \mathrm{~d}$. A comparison of the number of correct choices revealed no group difference $\left(F_{(2,26)}=1.6 ; p>0.1\right)$ and no effect of block $(F<1)$. To determine whether this increase in proactive interference resulted in poorer performance, the percentage of correct responses for sessions 9-12 of standard T-maze was compared with the percentage of correct responses for continuous alternation. There was a highly significant effect of condition $\left(F_{(1,26)}=\right.$ $101.7 ; p<0.0001)$, because the performance of all groups was worse when given massed trials but no group $\times$ condition interaction $\left(F_{(2,26)}=1.3 ; p>0.2\right)$.

The third condition assessed continuous alternation performance using delays of 10, 20, and $40 \mathrm{sec}$ between trials (Fig. 4). An ANOVA using factors group, day, and delays revealed no effect of group $(F<1)$ but a significant effect of delay $\left(F_{(2,52)}=\right.$ 12.6; $p<0.001)$ and day $\left(F_{(3,78)}=13.0\right.$; $p<0.001)$. This showed that the performance of all three groups deteriorated at longer delays but improved over training.

\section{Experiment 2: radial-arm maze}

For the radial-arm maze both total errors made and number of correct entries in the first eight choices were analyzed. When both measures gave the same pattern of results, only analyses using error scores are given. The first $14 \mathrm{~d}$ (stage 1) of the radial-arm maze task were analyzed in blocks of $2 \mathrm{~d}$. There were significant main effects of group $\left(F_{(2,26)}=7.2 ; p<0.005\right)$ (Fig. 5) and block $\left(F_{(, 156)}=16.0 ; p<\right.$ $0.0001)$. Subsequent comparisons using the Newman-Keuls test revealed that overall the MTTx animals were impaired relative to the shams $(p<0.01)$ and MBx animals $(p<0.05)$, but the MBx animals were not significantly different from the shams. An analysis of sequential choice responses showed no group difference $\left(F_{(2,26)}=1.4 ; p>0.2\right.$; means $\pm \mathrm{SE}, 1.54 \pm 0.1,1.38 \pm 0.1$, and $1.22 \pm 0.1$ for MTTx, MBx, and sham, respectively). To determine whether any transient impairment occurred at the initial stages of testing, the number of errors made in the first $4 \mathrm{~d}$ was analyzed separately. This revealed a significant effect of group $\left(F_{(2,26)}=4.1 ; p<0.05\right)$, and subsequent comparisons using the Newman-Keuls revealed that the MBx animals were significantly worse than the shams $(p<0.05)$, but no other comparisons were significant.

Stage 2 involved trials with a rotation of the maze after the first four choices to tax the use of extra maze cues. When the last $4 \mathrm{~d}$ of stage 1 (standard task) were compared with the $4 \mathrm{~d}$ of rotation, there was both a significant group difference $\left(F_{(2,26)}=9.7 ;, p<\right.$ 0.001 ; Fig. 6) and a group $\times$ condition interaction $\left(F_{(2,26)}=4.5\right.$; $p<0.05)$. Subsequent analyses of the simple effects showed a significantly greater disruptive effect of rotation on both the MTTx and MBx groups (both $p<0.05$ ). This is in contrast to the shams; their performance did not differ between the standard task and rotation (Fig. 6).

Stage 3 also involved maze rotation after the first four choices, but in addition there was a 20 min delay before the animals were returned to the maze. When analyses compared performance with the preceding $4 \mathrm{~d}$ of standard rotation, there was an overall effect of group $\left(F_{(2,26)}=6.0 ; p<0.01\right)$ (Fig. 6). There was also a 


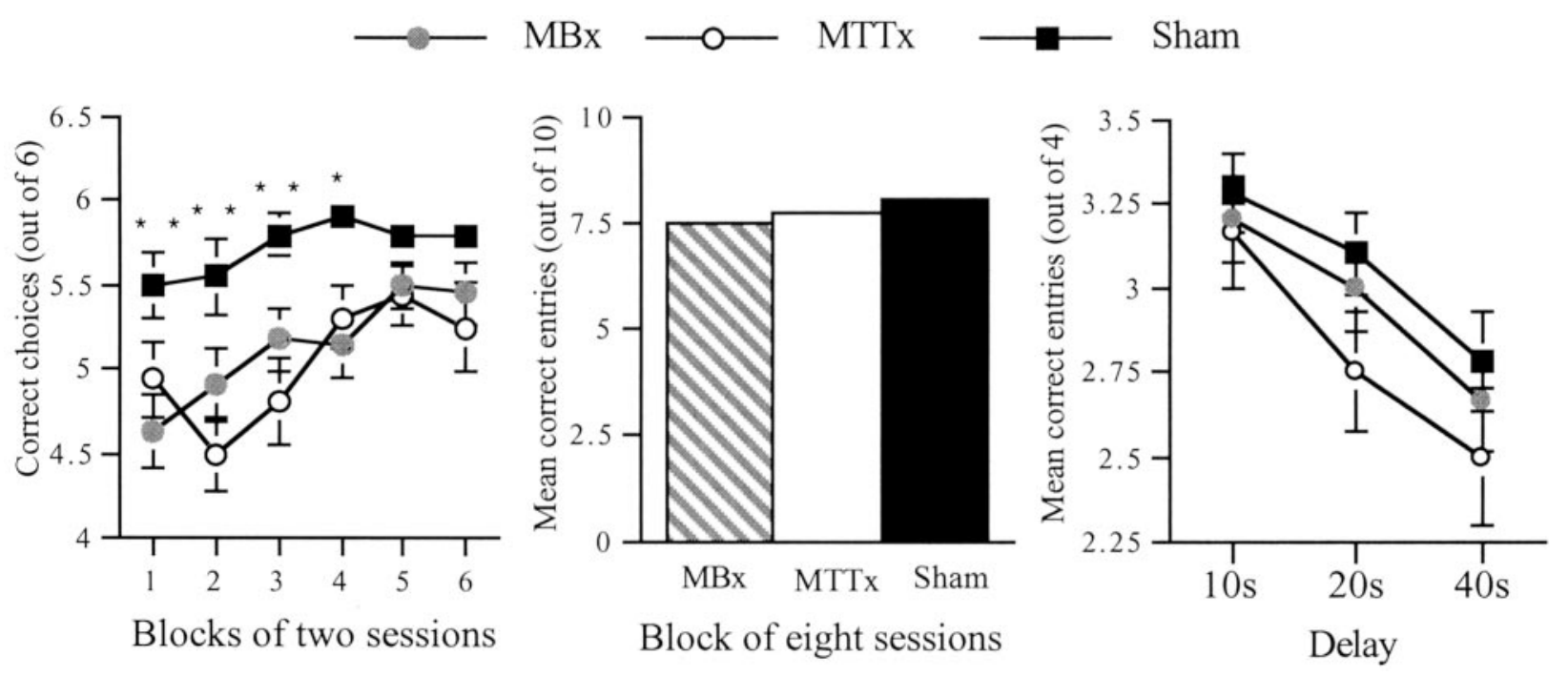

Figure 4. Reinforced alternation in the T-maze (experiment 1). The left-hand graph shows the scores over blocks of two sessions during task acquisition. The middle graph shows the scores of each group over the eight sessions of continual alternation. The right-hand graph shows the scores of each group when tested over each of three retention intervals. Data are shown as means \pm SEMs; where SE is very small, it is not visible on the graph. Significance of group differences: ${ }^{*} p<0.05,{ }^{* *} p<0.01$.

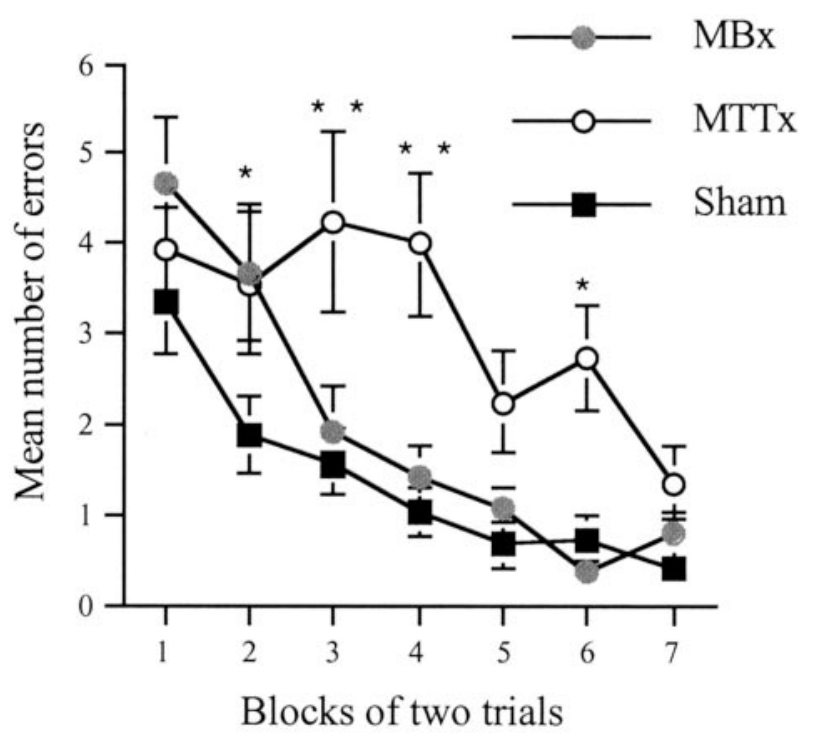

Figure 5. Standard radial-arm maze task (experiment 2). Number of errors during acquisition of the standard radial-arm maze task (stage 1). Data are shown as mean $\pm \mathrm{SE}$; where $\mathrm{SE}$ is very small, it is not visible on the graph. Significance of group differences: ${ }^{*} p<0.05$, ${ }^{* *} p<0.005$.

significant group $\times$ task interaction $\left(F_{(2,26)}=8.7 ; p<0.005\right)$ because the only group difference was during stage 2 (no delays). In fact, the MTTx lesion group was significantly less affected by the delay $(p<0.05)$. This paradoxical improvement in performance when delays were added presumably arose from the especially poor performance on stage 2. Post hoc analyses (NewmanKeuls) showed that only the MTTx and sham groups were significantly different $(p<0.01)$, although analyses using correct entries revealed that both lesion groups differed from the shams (both $p<0.05$ ).

Stage 4 involved the rats performing two consecutive trials each day for $4 \mathrm{~d}$. This was intended to increase proactive interference. An ANOVA was performed using group, day, and trial as factors. There was no group difference $\left(F_{(2,26)}=2.1 ; p>0.1\right)$, but there was a significant effect of trial $\left(F_{(1,26)}=70.6 ; p<0.001\right)$

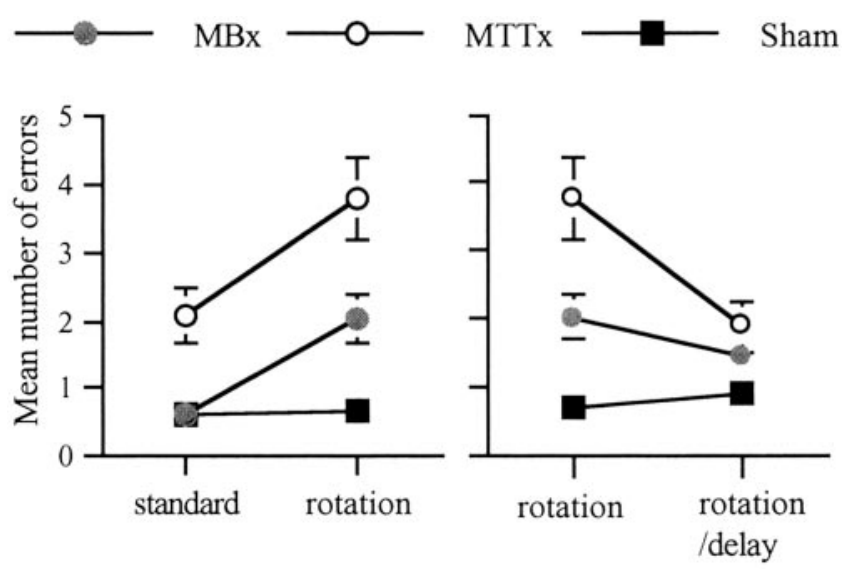

Figure 6. Radial-arm maze task (experiment 2). The left-hand graph shows the number of errors for the last four trials of the standard task compared and four trials with maze rotation. The right-hand graph shows the number of errors for $4 \mathrm{~d}$ of standard maze rotation and $4 \mathrm{~d}$ of maze rotation with 20 min delays. Data are shown as mean $\pm \mathrm{SE}$; where SE is very small, it is not visible on the graph.

with all groups making more errors on the second trial, presumably reflecting the increase in proactive interference. When looking at correct entries, there was also a significant group $\times$ day interaction $\left(F_{(6,78)}=2.5 ; p<0.05\right)$ because the MTTx animals performed significantly worse on the first day of stage $4(p<$ 0.005).

Stage 5 also involved rats performing two trials per day, but now the same maze was placed in a novel room. There was a significant group effect $\left(F_{(2,26)}=6.7 ; p<0.005\right)$ (Fig. 7). Subsequent comparisons using the Newman-Keuls test revealed a significant difference between the MTTx animals and both shams and MBx animals (both $p<0.05$ ) but no difference between the $\mathrm{MBx}$ animals and shams. There was also a group $\times$ trial interaction $\left(F_{(2,26)}=4.0 ; p<0.05\right)$, with only the MTTx group showing a significant effect of trial $(p<0.05)$. This was attributable to an improvement in the performance of MTTx animals on trial 2, and presumably reflects their very poor initial performance.

Because many animals showed very rapid learning of the new 


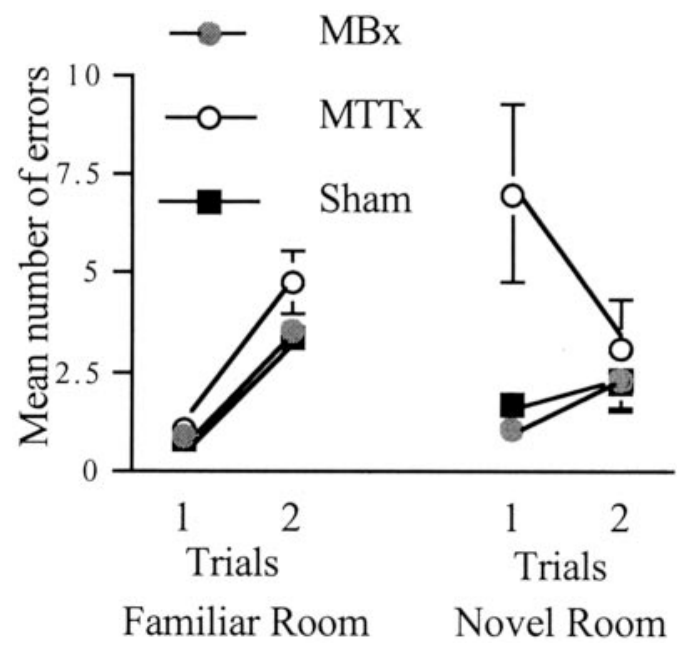

Figure 7. Radial-arm maze task (experiment 2). Shown are the number of errors when animals are given two trials per day for $4 \mathrm{~d}$ in the familiar room and $1 \mathrm{~d}$ in the novel room (see Materials and Methods). Data are shown as mean $\pm \mathrm{SE}$; where $\mathrm{SE}$ is very small, it is not visible on the graph.

room, a further set of comparisons was carried out using just trial 1 in the novel context and trial 1 of the last day of stage 4 . Using total errors, there was a significant effect of group $\left(F_{(2,26)}=7.0\right.$; $p<0.005)$ as well as room $\left(F_{(1,26)}=9.8 ; p<0.005\right)$. In addition there was a group $\times$ room interaction $\left(F_{(2,26)}=8.0 ; p<0.005\right)$ attributable to the MTTx animals' performance being significantly worse in the novel room.

\section{Experiment 3: water maze}

The first $12 \mathrm{~d}$ involved the standard working memory task in the water maze, with a $15 \mathrm{sec}$ ITI between the sample (trial 1) and test (trial 2) as well as the remaining two trials. There are some differences between path length and latency measures, in particular for the MB animals. They often swam very slowly on trial 1 so that the trial would time out. This resulted in high escape latencies but low path lengths. For this reason, both path length and latency data have been provided.

The $12 \mathrm{~d}$ of acquisition were blocked in groups of three, and analyses were performed using factors group, block, and trial. Analysis of the latency to reach the hidden platform revealed a significant group difference $\left(F_{(2,25)}=7.9 ; p<0.005\right)$. Subsequent comparisons using the Newman-Keuls test revealed a significant difference between both lesion groups and the shams (both $p<$ 0.01 ) but no difference between the MTTx and MBx animals. There was also a significant effect of block showing a general decrease in overall latency over the training period $\left(F_{(3,75)}=8.4\right.$; $p<0.001$ ) (Fig. 8). There was also a significant effect of trial $\left(F_{(3,75)}=63.2 ; p<0.001\right)$ showing some improvement by all groups over the trials (Fig. 8). Although there was no group $\times$ trial interaction $(p>0.1)$, analysis of the simple effects revealed no group difference at trial $1(p>0.1)$ but significant group differences at trials 2-4 (all $p<0.01$ ).

Analysis using path lengths for the $12 \mathrm{~d}$ of acquisition also revealed a significant effect of group $\left(F_{(2,25)}=6.4 ; p<0.01\right)$. Subsequent comparisons using the Newman-Keuls test revealed a significant difference between the MTTx and sham groups $(p<$ 0.01 ), but none of the other comparisons reached significance. There was also a significant effect of block showing a general decrease in overall path length over the training period $\left(F_{(3,75)}=\right.$ 37.3; $p<0.001$ ) (Fig. 8). There was also a significant main effect

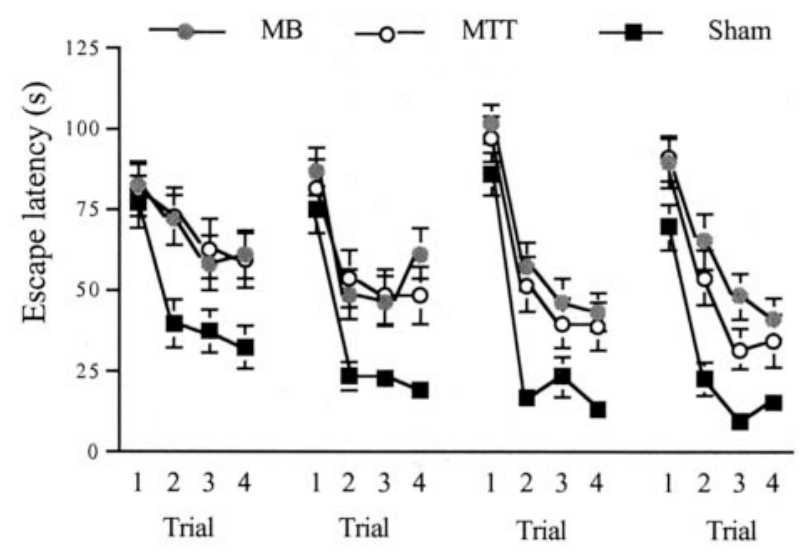

Blocks of three sessions

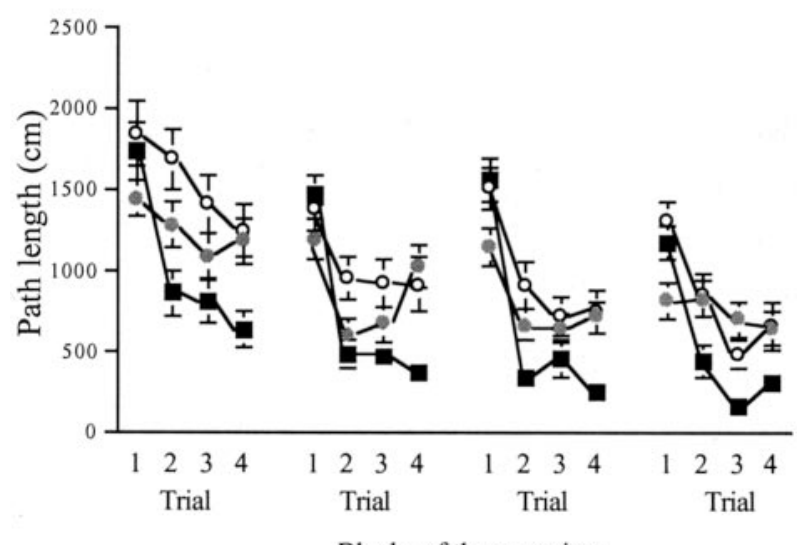

Figure 8. Water maze (experiment 3): standard working memory task in the water maze. Top graph shows escape latency to find hidden platform, and bottom graph shows path length. Data are shown as mean $\pm \mathrm{SE}$; where $\mathrm{SE}$ is very small, it is not visible on the graph.

of trial $\left(F_{(3,75)}=40.6 ; p<0.001\right)$ as well as a significant group $\times$ trial interaction $\left(F_{(6,75)}=4.9 ; p<0.005\right)$. The simple effects revealed group difference at all four trials $(p<0.05, p<0.005$, $p<0.05$, and $p<0.005$ for trials $1-4$, respectively). Only the sham group showed a significant shortening of path length across trials $(p<0.001)$ (Fig. 8).

The next stage (days 13-16) involved a 30 min delay between trial 1 (sample) and trial 2 (working memory test), with the remaining two trials run as before (Fig. 9).

To determine the effect of delay on performance, the last block from the first stage (days 10-12) was compared with the block of delay trials. Because the delay component was between trials 1 and 2, with the remainder of the trials being run as normal, only trials 1 and 2 were included in the analysis. Using latency there was a significant effect of group $\left(F_{(2,25)}=8.9 ; p<0.005\right)$ and trial $\left(F_{(2,25)}=83.4 ; p<0.001\right)$, but not of delay condition $\left(F_{(1,25)}=\right.$ $3.4 ; p=0.08)$. When path lengths were used, as well as there being significant effects of group $\left(F_{(2,25)}=3.5 ; p<0.05\right)$ and trial $\left(F_{(1,25)}=37.9 ; p<0.001\right)$, there was a significant effect of delay condition $\left(F_{(1,25)}=5.3 ; p<0.05\right)$ but no group $\times$ delay condition interaction $(F<1)$.

Days 17 and 18 involved the standard working memory procedure again. Days 19 and 20 also involved this procedure but this time in a novel water maze. All $4 \mathrm{~d}$ were included in an ANOVA with group, room, day, and trial as factors. An analysis using escape latencies revealed a significant effect of group $\left(F_{(2,25)}=\right.$ 8.6; $p<0.005)$, and simple effects revealed a significant group 


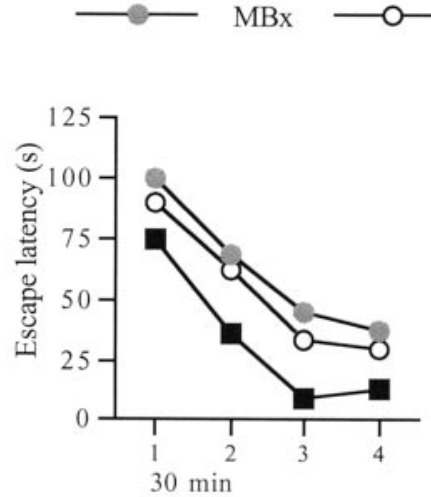

Block of four sessions

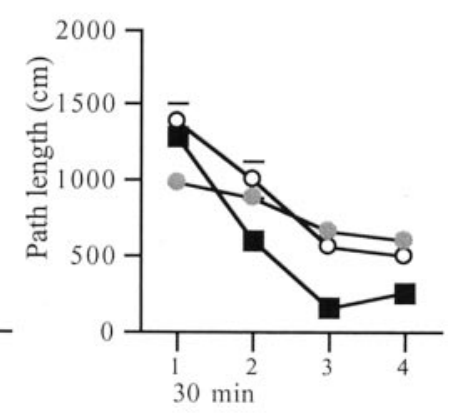

Block of four sessions
Figure 9. Water maze (experiment 3): working memory task with 30 min delays between trials 1 and 2. Graphs show escape latencies (left) and path lengths (right). Data are shown as mean $\pm S E$; where $S E$ is very small it is not visible on the graph.

difference at trial $1(p<0.05)$, trial $2(p<0.001)$, and trial 3 $(p<0.05)$ but not trial $4(p>0.1)$. Comparisons using the Newman-Keuls test again showed a significant difference between MTTx and sham $(p<0.05)$ and MB and sham $(p<0.01)$ but not between the MBx and MTTx groups. There was also a significant effect of trial $\left(F_{(3,75)}=86.8 ; p<0.001\right)$. There was no effect of room $\left(F_{(1,25)}=2.4 ; p>0.1\right)$ or room $\times$ group interaction $(F<1)$.

When the $2 \mathrm{~d}$ in the familiar room were compared with the $2 \mathrm{~d}$ in the novel room using path length, there was again a significant effect of group $\left(F_{(2,25)}=6.0 ; p<0.01\right)$ (Fig. 10). Subsequent comparisons revealed significant differences only between the MTTx and sham groups $(p<0.01)$. There was also a significant effect of room $\left(F_{(1,25)}=15.1 ; p<0.001\right)$, with greater overall path lengths in the novel room. There was a significant effect of trial $\left(F_{(3,75)}=55.6 ; p<0.001\right)$ as well as a significant group $\times$ trial interaction $\left(F_{(6,75)}=2.6 ; p<0.05\right)$. Subsequent examination of the simple effects revealed significant group differences at trial $1(p<0.05)$ and trial $2(p<0.005)$ but not at trials 3 and 4 (both $p>0.05$ ). Only MTT and sham groups showed significant shortening of path length across trials (both $p<0.005$ ).

Because the first two trials in the novel room are likely to be the most sensitive to the demands of the new environment, trials 1 and 2 of the last day in the familiar room (day 18) were compared with trials 1 and 2 in the novel room (day 19). There was a significant effect of room $\left(F_{(1,25)}=4.3 ; p<0.05\right)$ but no group $\times$ room interaction $\left(F_{(2,25)}=1.4 ; p<0.1\right)$. There was also a significant group $\times$ trial interaction $\left(F_{(2,25)}=5.0 ; p<0.05\right)$ because there was only a group difference at trial $2(p<0.05)$, and the shams were the only group to show significant shortening of path length over the two trials $(p<0.005)$.

\section{Discussion}

The present study examined the effects of lesions in two connected components of the Delay and Brion circuit on three tasks of spatial memory. Animals with MTT or MB lesions were trained on working memory tasks in the T-maze, radial-arm maze, and water maze. Although rats with MB lesions have been tested before on the standard versions of these three tasks, rats with MTT lesions have been tested only on T-maze alternation. In addition to the standard versions of these tasks, the study then examined manipulations including massing trials to increase levels of proactive interference, increasing retention intervals, and taxing the rapid processing of new, extra-maze stimuli. In this way, performance was assessed against three current hypotheses of MB function.

Both MB and MTT lesions impaired the acquisition of all three spatial tasks. A transient T-maze alternation impairment was found for both lesion groups, consistent with previous studies of MB (Aggleton et al., 1990, 1995) and MTT (Field et al., 1978; Thomas and Gash, 1985) lesions. For the radial-arm maze task, the MTTx group showed the more persistent deficit, because the MBx animals were impaired only for the first few sessions. With training, both lesion groups improved on the radial-arm maze task, but subsequent control manipulations (maze rotation) showed that neither the MBx nor MTTx animals performed the task in the same way as the sham group. This reveals a failure to use extra-maze cues effectively and suggests that the transient impairments hid a more permanent abnormality in spatial learning. Likewise, Neave et al. (1997) found that rats with MB lesions performing T-maze alternation remained less reliant on extramaze cues than control groups. These apparent failures to use allocentric cues also accord with the matching-to-place task in the water maze because persistent deficits were found in this and a previous lesion study (Santin et al., 1999). Although other studies of $\mathrm{MB}$ lesions have also found relatively permanent spatial working memory deficits (Saravis et al., 1990; Sziklas and Petrides, 1993; Neave et al., 1997), these are more difficult to interpret because in all of these cases the supramammillary nuclei were included in the lesion.

To test the first hypothesis, that the MB help to distinguish separate events by enhancing contextual difference, the level of proactive interference was increased in the T-maze and radialarm maze. In the T-maze, this involved giving rats massed trials (continuous alternation), and in the radial-arm maze they were given two consecutive trials. For both tasks, raising interference led to a significant decrease in overall levels of accuracy. Although this is consistent with a disruptive effect caused by increased interference, there are other factors such as fatigue or loss of motivation that could have contributed. Nevertheless, neither lesion group was differentially sensitive to this manipulation and performed at control levels. Although a previous study found that MB-lesioned animals were impaired on continuous alternation in the T-maze (Aggleton et al., 1995), other studies have failed to find $\mathrm{MB}$ lesion deficits on automated spatial tasks (e.g., delayed non-matching to a sample lever) that have a high degree of proactive interference (Aggleton et al., 1991; Harper et al., 1994). There is evidence, however, that T-maze alternation and the automated non-matching task make qualitatively different spatial demands, although they can both be characterized as nonmatching to a spatial sample. For example, double dissociations are found between the effects of prefrontal and cingulum bundle lesions on these two tasks (Aggleton et al., 1995). For this reason, the present results generalize our understanding of the contribution of the MB beyond that found for automated tasks.

The second hypothesis was that MB lesion animals are less able to hold information over longer intervals, thereby predicting greater impairments with longer retention intervals (Sziklas and Petrides, 1993; Beracochea and Jaffard, 1995). Delays of 10, 20, and $40 \mathrm{sec}$ were included in the T-maze task, 20 min delays were included in the radial-arm maze task, and 30 min delays were included in the water maze task. In none of these conditions were the two lesion groups differentially impaired with longer delays. Similarly, tests of automated matching and non-matching-tosample failed to find $\mathrm{MB}$ lesion deficits with delays of up to $64 \mathrm{sec}$ (Aggleton et al., 1991; Harper et al., 1994). Likewise, previous 

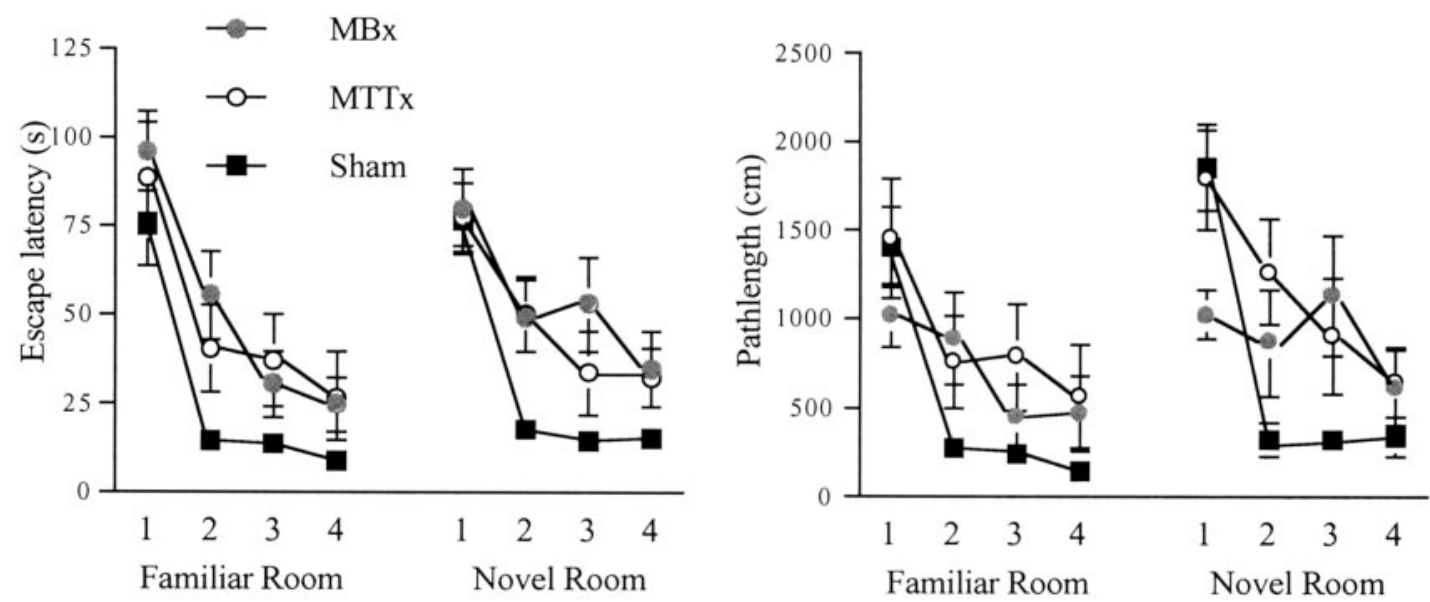

Figure 10. Water maze (experiment 3): last day of working memory task in familiar room and the first day of the same task in a novel room. Graphs show escape latencies (left) and path lengths (right). Data are shown as mean $\pm \mathrm{SE}$; where $\mathrm{SE}$ is very small, it is not visible on the graph.

T-maze alternation studies have reported intact performance with increasing retention delays (Aggleton et al., 1990) [but see Aggleton et al. (1995) and Beracochea and Jaffard (1995)]. Finally, using shorter intervals (30 sec and $5 \mathrm{~min}$ ), Santin et al. (1999) also found that MB lesion animals were not disproportionately impaired by longer delays when matching-to-place in a water maze. Thus, although the present study looked at a wider variety of tasks and longer retention intervals than used previously, there was still no evidence of specific delay-dependent deficits.

The third hypothesis was that the MBs are needed when rapidly encoding new spatial information. Evidence for this came from the initial acquisition deficit in experiments 1 and 2 and from the finding that structures in the Delay and Brion circuit are activated when animals are required to perform the standard radial-arm maze task in a novel room (Vann et al., 2000a,b). For this reason, rats in the present study were moved to a novel room and given two consecutive trials in the radial-arm maze. Performance was compared with that in the familiar room. Although neither the sham nor MBx group was affected by the room switch, the MTTx animals were significantly impaired on the first trial but recovered by the second trial. This suggests that it took them longer to encode these new stimuli to perform the task effectively. The results from moving to a water maze in a new room are less clear, however. Although there was an overall effect of moving rooms, the lesion groups were not differentially disrupted. This comparison is limited, however, by the fact that the MBx and MTTx groups remained impaired up to the room switch condition, so previous performance could not be matched with the sham animals. Furthermore, because the task itself requires the rapid learning of a new location on every session, an additional impairment might not necessarily be detected.

For the majority of tasks, the MBx and MTTx animals performed at equivalent levels. This highlights the critical importance of the MTT for MB function. The only differences were during acquisition of the radial-arm maze task and the transfer of the same task to a novel room. In both cases the MTTx group showed the greater impairment. The discreteness of the MTT lesions suggests that these greater deficits were not caused by the involvement of additional fiber pathways. In this context it should also be noted that the supramammillary nuclei do not project through the MTT (Vertes, 1992). The most probable explanation, therefore, is that the MTT lesions produced the more complete disconnection of the Delay and Brion circuit, because the neurotoxic injections always left some spared cells.

That the MB contribute to spatial memory is expected, not only because of their central anatomical placement between the hippocampus and anterior thalamic nuclei, but also from the presence of theta-responsive cells (Kocsis and Vertes, 1994; Kirk et al., 1996) and head-direction cells in this region (Blair et al., 1998; Stackman and Taube, 1998). These theta-responsive cells are thought to relay the theta-rhythmic signal to other parts of the limbic system (Bland et al., 1995) but do not have a role in generating theta in the hippocampus or septum (Kirk et al., 1996). The reciprocal loop between MB and the tegmental nuclei of Gudden may serve to further process information from the hippocampus to the anterior thalamic nuclei by adding vestibular information (Blair et al., 1998) and so aid encoding. This is because the dorsal tegmental nucleus contains both head-direction and angular velocity cells that could work in concert with headdirection cells in the lateral mammillary nucleus (Sharp et al., 2001). The potential importance of this reciprocal link with Gudden's tegmental nuclei is suggested by the disruptive effect of mammillotegmental tract lesions on T-maze alternation (Field et al., 1978). Like the MB, the ventral tegmental nuclei of Gudden show theta rhythm activity (Kocsis et al., 2001), which is thought to derive from hippocampal activity.

Neither the MB nor MTT lesions appeared to result in degeneration or cell loss in the anterior thalamic nuclei in the present study, although MTT lesions have been shown to attenuate training-induced activity in the anteroventral thalamic nucleus as well as baseline activity in this region (Gabriel et al., 1995). Lesions of the lateral mammillary nuclei have also been shown to result in anterodorsal head-direction cells losing their directional firing properties (Blair et al., 1998, 1999). However, although MTT transection or MB lesions will disconnect the anterior thalamic nuclei by cutting the Delay and Brion connection, there remains the direct fornical route from the hippocampus to the anterior thalamic nuclei. This projection may well explain why MB lesions have not proved to be as disruptive as anterior thalamic lesions (Aggleton et al., 1991, 1995). Nevertheless, the present study shows that disconnecting the Delay and Brion circuit does impair the learning of new spatial tasks but need not increase sensitivity to proactive interference or delay. This pattern of results points to an encoding deficit for spatial memory tasks that includes learning new locations in familiar settings. The 
nature of this encoding deficit cannot be defined on the basis of the present study, but other findings point to a likely failure to use directional heading signals effectively (Blair et al., 1998, 1999). This may well result in the reliance of other, less effective strategies that are most apparent when there is a premium on rapid encoding.

\section{References}

Aggleton JP, Brown MW (1999) Episodic memory amnesia, and the hippocampal-thalamic axis. Behav Brain Sci 22:425-444.

Aggleton JP, Hunt PR, Shaw C (1990) The effects of mammillary body and combined amygdalar-fornix lesions on tests of delayed non-matching-tosample in the rat. Behav Brain Res 40:145-157.

Aggleton JP, Keith AP, Saghal A (1991) Both fornix and anterior thalamic, but not mammillary, lesions disrupt delayed non-matching-to-position memory in rats. Behav Brain Res 44:151-161.

Aggleton JP, Neave N, Nagle S, Hunt PR (1995) A comparison of the effects of anterior thalamic, mammillary body and fornix lesions on reinforced spatial alternation. Behav Brain Res 68:91-191.

Allen GV, Hopkins DA (1990) Topography and synaptology of mammillary body projections to the mesencephalon and pons in the rat. J Comp Neurol 301:214-231.

Beracochea DJ, Jaffard R (1995) The effects of mammillary body lesions on delayed matching and delayed non-matching to place tasks in the mice. Behav Brain Res 68:45-52.

Blair HT, Cho J, Sharp PE (1998) Role of the lateral mammillary nucleus in the rat head direction circuit: a combined single unit recording and lesion study. Neuron 21:1387-1397.

Blair HT, Cho J, Sharp PE (1999) The anterior thalamic head-direction signal is abolished by bilateral but not unilateral lesions of the lateral mammillary nucleus. J Neurosci 19:6673-6683.

Bland BH, Konopacki J, Kirk IJ, Oddie SD, Dickson CT (1995) Discharge patterns of hippocampal theta-related cells in the caudal diencephalon of the urethane-anesthetized rat. J Neurophysiol 74:322-333.

Cruce JAF (1975) An autoradiographic study of the projections of the mammillothalamic tract in the rat. Brain Res 85:211-219.

Delay J, Brion S (1969) Le syndrome de Korsakoff. Paris: Masson and Cie.

Dusoir H, Kapur N, Byrnes DP, McKinstry S, Hoare RD (1990) The role of diencephalic pathology in human memory disorder. Brain 113:1695-1706.

Ennaceur A, Aggleton JP (1997) The effects of neurotoxic lesions of the perirhinal cortex combined to fornix transection on object recognition in the rat. Behav Brain Res 88:181-193.

Field TD, Rosenstock J, King C, Greene E (1978) Behavioral role of the mammillary efferent system. Brain Res Bull 3:451-456.

Gabriel M, Cuppernell C, Shenk JI, Kubota Y, Henzi V, Swanson D (1995) Mammillothalamic tract transection blocks anterior thalamic traininginduced neuronal plasticity and impairs discriminative avoidance behavior in rabbits. J Neurosci 15:1437-1445.

Gaffan D (1992) The role of the hippocampus-fornix-mammillary system in episodic memory. In: Neuropsychology of memory (Squire LR, Butters N, eds), pp 336-346. New York: Guildford.

Gamper E (1928) Zur Frage der Polioencephalitis der chronischen Alkoholiker. Anatomische Befunde beim chronischem Korsakow und ihre Beziehungen zum klinischen Bild. Deutsche Z Nervenheilkd 102:122-129.

Harper DN, McLean AP, Dalrymple-Alford J (1994) Forgetting in rats following medial septum or mammillary body damage. Behav Neurosci 198:1-12.

Hildebrandt H, Muller S, Bussmann-Mork B, Goebel S, Eilers N (2001) Are some memory deficits unique to lesions of the mammillary bodies? J Clin Exp Neuropsychol 23:490-501.

Jaffard R, Beracochea D, Cho Y (1991) The hippocampal-mamillary system: anterograde and retrograde amnesia. Hippocampus 1:275-278.

Kirk IJ (1998) Frequency modulation of hippocampal theta by the supramammillary nucleus, and other hypothalamo-hippocampal interactions: mechanisms and functional implications. Neurosci Biobehav Rev 22:291-302.

Kirk IJ, Oddie SD, Konopacki J, Bland BH (1996) Evidence for differential control of posterior hypothalamic, supramammillary, and medial mammillary theta-related cellular discharge by ascending and descending pathways. J Neurosci 16:5547-5554.

Kocsis B, Vertes RP (1994) Characterization of neurons of the supramammillary nucleus and mammillary body that discharge rhythmically with hippocampal theta in the rat. J Neurosci 14:7040-7052.

Kocsis B, Viano Di Prisco G, Vertes RP (2001) Theta synchronization in the limbic system: the role of Gudden's tegmental nuclei. Eur J Neurosci 13:381-388.

Krieckhaus EE, Randall D (1968) Lesions of the mammillothalamic tract in rat produce no decrements in recent memory. J Neurol 91:369-378.

Morris RGM, Garrud P, Rawlins JNP, O’Keefe J (1982) Place navigation impaired in rats with hippocampal lesions. Nature 297:681-683.

Morris RGM, Schenk F, Tweedie F, Jarrard LE (1990) Ibotenate lesions of hippocampus and/or subiculum: dissociating components of allocentric spatial learning. Eur J Neurosci 2:1016-1028.

Neave N, Nagle S, Aggleton JP (1997) Evidence for the involvement of the mammillary bodies and cingulum bundle in allocentric spatial processing by rats. Eur J Neurosci 9:941-955.

O'Keefe J, Nadel L (1978) The hippocampus as a cognitive map. Oxford: Oxford UP.

Olton DS, Samuelson RJ (1976) Remembrance of places passed: spatial memory in rats. J Exp Psychol Anim Behav Proc 2:97-116.

Olton DS, Walker JA, Gage FH (1978) Hippocampal connections and spatial discrimination. Brain Res 139:295-308.

Paxinos G, Watson C (1997) The rat brain in stereotaxic coordinates. San Diego: Academic.

Santin LJ, Rubio S, Begaga A, Arias JL (1999) Effects of mammillary body lesions on spatial reference and working memory tasks. Behav Brain Res 102:137-150.

Saravis S, Sziklas V, Petrides M (1990) Memory for places and the region of the mammillary bodies in the rat. Eur J Neurosci 2:556-564.

Seki M, Zyo K (1984) Anterior thalamic afferents from the mammillary body and the limbic cortex in the rat. J Comp Neurol 229:242-256.

Sharp PE, Tinkelman A, Cho J (2001) Angular velocity and head directions signals recorded from the dorsal tegmental nucleus of Gudden in the rat: implications for path integration in the head direction cell circuit. Behav Neurosci 115:571-588.

Stackman RW, Taube JS (1998) Firing properties of rat lateral mammillary single units: head direction, head pitch, and angular head velocity. J Neurosci 18:9020-9037.

Sutherland RJ, Rodriguez AJ (1989) The role of the fimbria/fornix and some related subcortical structures in place learning and memory. Behav Brain Res 32:265-277.

Sziklas V, Petrides M (1993) Memory impairments following lesions to the mammillary region of the rat. Eur J Neurosci 5:525-540.

Sziklas V, Petrides M (1998) Memory and the region of the mammillary bodies. Prog Neurobiol 54:55-70.

Tanaka Y, Miyazawa Y, Akaoka F, Yamada T (1997) Amnesia following damage to the mammillary bodies. Neurology 48:160-165.

Thomas GJ, Gash DM (1985) Mammillothalamic tracts and representational memory. Behav Neurosci 99:621-630.

Vann SD, Brown MW, Aggleton JP (2000a) Fos expression in the rostral thalamic nuclei and associated cortical regions in response to different spatial memory tests. Neuroscience 101:983-991.

Vann SD, Brown MW, Erichsen JT, Aggleton JP (2000b) Fos imaging reveals differential patterns of hippocampal and parahippocampal subfield activation in rats in response to different spatial memory tests. J Neurosci 20:2711-2718.

Vertes RP (1992) PHA-L analysis of projections from the supramammillary nucleus in the rat. J Comp Neurol 326:595-622.

Victor M, Adams RD, Collins GH (1989) The Wernicke-Korsakoff syndrome and related neurologic disorders due to alcoholism and malnutrition. Philadelphia: F. A. Davis.

Watanabe K, Kawana E (1980) A horseradish peroxidase study on the mammillothalamic tract in the rat. Acta Anat 198:394-401. 